\title{
Relationship of Hospital Star Ratings to Race, Education, and Community Income
}

\author{
Bo Shi, PhD', Christopher J King, PhD², Sean Shenghsiu Huang, PhD ${ }^{2 \star}$
}

'Department of Accounting and Finance, Elmer R Smith College of Business and Technology, Morehead State University, Morehead, Kentucky; ${ }^{2}$ Department of Health Systems Administration, Georgetown University, Washington, District of Columbia.

INTRODUCTION: The Centers for Medicare \& Medicaid Services (CMS) publishes hospital quality ratings to provide more transparent and useable quality information to patients and stakeholders. However, there is a gap in the literature regarding the geographic distribution of the hospitals with higher star ratings. In this paper, we focus on the associations between star ratings and community characteristics, including racial/ethnic mix, household income, educational attainment, and regional difference.

METHODS: A retrospective study and cross-sectional logistic and multinomial logistic regression analyses.

RESULTS: According to the multivariate regression results, hospitals in areas with lower income, lower educational attainment, and higher minority population shares have lower quality ratings (lower income: odds ratio [OR] 0.67;
95\% Cl, 0.49-0.91; lower education: OR 0.66; 95\% Cl, 0.51-0.85; higher minority: OR 0.52; 95\% $\mathrm{Cl}, 0.40-0.69$ ). Compared with hospitals in the Midwest, hospitals in Northeast, South, and West regions have lower quality ratings (Northeast: OR 0.37; 95\% Cl, 0.25-0.56; South: OR 0.68 ; $95 \% \mathrm{Cl}, 0.51-0.91$; West: OR 0.69; $95 \% \mathrm{Cl}, 0.49-0.97)$.

DISCUSSION AND CONCLUSION: Overall, our results show that hospitals with higher star ratings are less likely to be located in communities with higher minority populations, lower income, and lower levels of educational attainment. Findings contribute to the discussion of integrating social factors in hospital quality star rating calculation methodologies. Journal of Hospital Medicine 2020;15:588-593. () 2020 Society of Hospital Medicine
$\mathrm{H}$

ospitals play important roles in the healthcare ecosystem. Currently, they account for approximately one-third of more than $\$ 3$ trillion dollars spent on healthcare annually. ${ }^{1}$ To contain costs, improve patient experience, and advance population health, there has been progress in standardizing quality metrics and increasing transparency around key performance metrics.

Launched in 2016, the Overall Hospital Quality Star Rating was developed by the Centers for Medicare \& Medicaid Services (CMS) as a means of assessing quality and outcome measures. More importantly, star ratings are aimed to enhance the usability and accessibility of information about quality. The rating system evaluates seven quality categories: mortality, safety, readmission, patient experience, effectiveness, timeliness, and efficient use of medical imaging. Hospitals that have at least three measures within at least three measure categories, including one outcome group (mortality, safety, or readmission) are eligible for an overall rating based on a five-star system. ${ }^{2}$

While the intent of quality ratings is to summarize high-di-

\footnotetext{
*Corresponding Authors: Sean Shenghsiu Huang, PhD; Email: Sean.Huang@ georgetown.edu; Telephone: 202-687-5494.

Published online first September 23, 2020.

Find additional supporting information in the online version of this article.
}

Received: June 18, 2019; Revised: January 31, 2020;

Accepted: February 1, 2020

() 2020 Society of Hospital Medicine DOI 10.12788/jhm.3393 mensional information to facilitate patients in choosing hospitals with better quality, it is unclear whether patients have equal geographic proximity to hospitals with high ratings. Although researchers have examined overall quality ratings by hospital type (community, specialty, teaching, bed size), ${ }^{3}$ there is an opportunity to expand the body of knowledge at the intersection of overall star rating and race/ethnicity, education attainment, income level, and geographic region.

This study complements prior investigations on the topic. For example, Osbourne et al found that comorbidities and socioeconomic barriers were leading factors in observed mortality disparities between Black and White patients. ${ }^{4}$ Since mortality ratings are factored into overall star ratings, hospitals that serve low-income communities of color with high-acuity volumes may be at risk for lower star quality ratings. Trivedi et al found that, compared with White patients, Black and Hispanic patients were more likely to use low-volume hospitals for cardiac procedures. In addition, Black patients experienced worse outcomes. ${ }^{5}$ Insurance barriers, limited access to specialty care providers, and residential segregation may explain the chasm. These factors, often beyond hospitals' control, may impact readmissions, which are also factored into overall quality ratings. Additionally, $\mathrm{Hu}$ and Nerenz found that, on average, the most "stressed" cities have lower quality ratings than less "stressed" cities. ${ }^{6}$ Stress markers include poverty, unemployment, divorce rate, and adult health conditions. Other findings suggest readmission rates are correlated with patient provider ratios, community characteristics, and poor social and economic conditions that influence 
decision-making. ${ }^{7-9}$ Some investigators have explored quality ratings in other sectors of healthcare. For example, residents in socioeconomically disadvantaged counties are less likely to access nursing homes with higher star ratings. ${ }^{9}$

In light of new and emerging value-based payment models, coupled with efforts to risk-adjust for socioeconomic conditions that may compromise desired outcomes, this study sought to expand the scope of knowledge by offering insight on the association between hospital quality ratings and socioeconomic factors and geographic indicators. Particularly, we focus on the minority population percentage, county-level household income, education, dual eligibility, rural/urban designation, and geographic region.

\section{METHODS}

\section{Data and Study Sample}

Our analysis relies on data extracted from multiple sources. We obtained hospital overall quality ratings from the Hospital Compare website (www.medicare.gov/hospitalcompare) released in July 2018. We also included key hospital characteristics extracted by American Hospital Directory and Medicare cost reports. Socioeconomic and demographic variables were obtained from the Area Health Resources Files (AHRF) maintained by Health Resources \& Services Administration. Hospital referral region data was downloaded from Dartmouth Atlas Project. We included only acute hospitals that were certified by CMS. Hospitals with missing overall star rating values were excluded. Our study included 3,075 acute care hospitals in 1,047 counties and 306 hospital referral regions.

\section{Dependent Variable: Hospital Quality Ratings}

Our main outcome variables are hospital quality ratings reported by CMS. The overall star ratings use 64 of more than 100 quality measures and ranges from one to five stars, with five stars representing the highest quality. Our study uses the hospital quality star rating released in July 2018. The measurement period starts in January 2014 and extends to September 2017. Because of space limitation, we only present the results on the overall rating. The full results of all seven quality domains are provided in appendices.

\section{Key Independent Variables}

Key variables of interest are the socioeconomic factors of the communities served by the hospital. Specifically, our analysis focuses on minority population percentage, household income, education attainment, Medicare/Medicaid dual eligibility, urban/rural designation, and geographic region. For these key variables except urban/rural designation and geographic region, we created categorical variables indicating whether the values are below the national median (low group), in the 3rd quartile (intermediate group), and in the 4th quartile (high group). Group cutoffs are based on socioeconomic and demographic variables reported by AHRF for all counties nationwide. Because we use the county averages as the cutoff values and each county has a different number of hospitals, the number of hospitals distributes unevenly in each quartile.
Additionally, we grouped the 1st and 2nd quartiles as the low group because there are fewer hospitals in these two quartiles. Education attainment is measured by the percentage of population above 25 years old with a college degree. "Hospital access" is defined as a measure for the availability of services from competing hospitals, and we counted the number of hospitals available in a hospital referral region. For the 306 hospital referral regions, the number of hospitals ranges from 1 to 71 with an average of 12 .

\section{Statistical Model}

To study the relationship between quality rating and socioeconomic factors, we used both logistic and multinomial logistic regression models. The regression model can be described as follows:

$$
\begin{aligned}
\mathrm{Q}_{i} & =\text { Minority }_{i} \beta_{1}+\text { Income } \\
& \beta_{2}+\text { Population Age } \beta_{3} \\
& + \text { Education }_{i} \beta_{4}+\text { Access }_{i} \beta_{5}+\text { Dual_Eligible } \beta_{6}+\text { Rural } \beta_{7}+\text { Region }_{i} \beta_{8} \\
& + \text { Hosp }_{i} \gamma+\epsilon_{i}
\end{aligned}
$$

In the logistic model, $Q_{i}$ represents the dependent variable indicating whether a hospital has an overall quality star rating of either one star or five stars; we also ran a multinomial logistic regression model in which the hospital overall quality star rating ranges from one star to five stars with one-star increments. These ordinal regression models include key socioeconomic factors, such as percentage of population that is a minority, the average household income, the education attainment level, access to hospitals, the percentage of population that is Medicare/Medicaid dual-eligible, and the rurality of a hospital. We also include a set of dummy variables to control for region differences. [Hosp] is a vector of hospital characteristics, including ownership status, teaching status, and hospital size.

To examine extreme hospital quality (ie, one or five stars) overall ratings in relation to socioeconomic factors of serving communities, we first used the logistic regression model to predict probabilities of hospitals with either one-star or fivestar ratings. We then compared the marginal probabilities of key socioeconomic factors. Finally, we treated the overall quality rating collectively, ranging from one to five stars, as an ordinal variable and applied multinomial logistic regression to produce odds ratios of relationship of key variables with higher quality rating hospitals. For all these models, standard errors are clustered at the hospital referral region level. Models are estimated by generalized estimating equations. Statistical analyses were conducted in SAS 9.2.

\section{RESULTS}

We first present the summary statistics of key variables in Table 1. The estimated marginal probabilities and odds ratios from the multivariate regressions are reported in Table 2.

\section{Distribution of Quality Ratings}

The distribution of hospital quality rating is shown in the Figure. About $8 \%$ of the hospitals received a one-star rating, whereas $9.95 \%$ of the hospitals had a five-star rating. Most of the hos- 


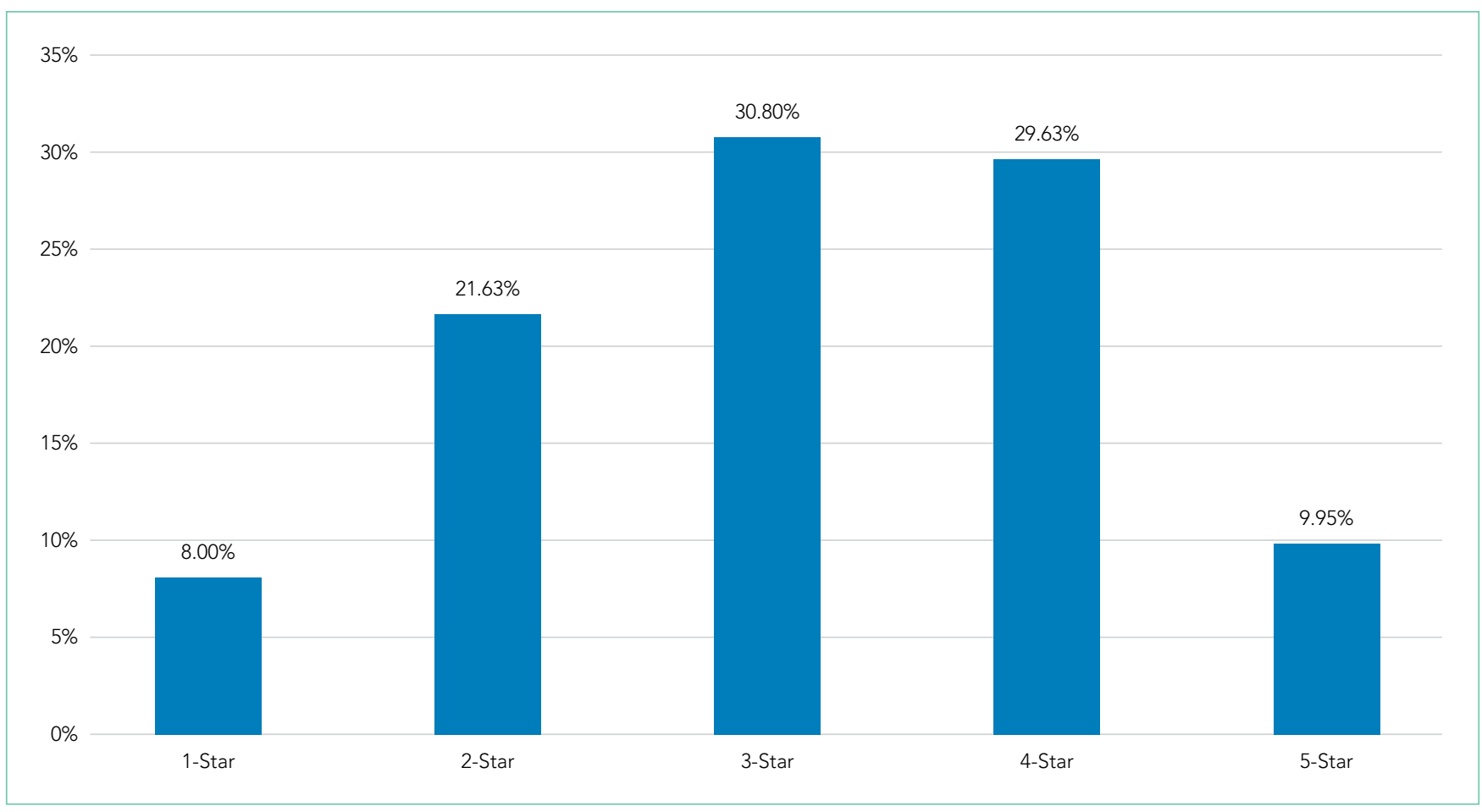

FIG. Hospital Overall Star Ratings Distribution

There are 3,075 acute care hospitals included in this study after merging four data sources: 246 hospitals are rated 1-star, 665 hospitals are rated 2-star, 947 hospitals are rated 3-star, 911 hospitals are rated 4-star, and 306 hospitals are rated 5-star. Most recent star rating can be found at https://www.medicare.gov/hospitalcompare/Data/Hospital-overall-ratings-calculation.htm

pitals received two, three, and four stars with frequencies of $21.63 \%, 30.80 \%$, and $29.63 \%$, respectively. The distribution of quality ratings with respect to socioeconomic and geographic factors are presented in Table 1. Most hospitals in our sample were located in counties where the minority population percentage was above the national median (8.21\%). The hospitals in counties with highest minority presence had a lower overall rating (2.86). There is a clear gradient between the median household income and hospital overall rating. About $43 \%$ of hospitals were in counties in which the median household income was in the 4 th quartile, whereas only $31 \%$ of hospitals are in counties with a median household income below the national median. Hospitals in counties with high income also have higher overall rating (3.24). In terms of urban/rural hospitals, there are more urban hospitals (70\%) but with a lower overall rating of 3.04 , compared with rural hospitals $(30 \%, 3.31)$. We also found that the counties with higher education attainment and lower dual-eligible population tend to have higher hospital ratings. Geographically, hospitals in the Midwest and West have higher average overall quality ratings than do those in the Northeast and South.

\section{Minority Population Percentage and Hospital Rating} As shown in Table 2, results from the logistic regression show that, compared with those in counties with low minority population percentage, hospitals in counties with high minority population percentage have higher marginal probabilities to have one-star ratings, and the result is statistically significant at the $1 \%$ level. At the same time, hospitals in counties with intermediate minority percentage have lower marginal probabilities of having a five-star rating. On the other hand, the odds ratio from the multinomial logistic regressions show that minority population percentage is negatively correlated with hospital rating, statistically significant at the $1 \%$ level.

\section{Median Household Income and Hospital Rating}

We found a statistically significant relationship between household income and hospital quality rating. Hospitals in lower income groups are more likely to have one-star ratings. The odds ratio analysis provides consistent evidence that higher household income is correlated with star ratings.

\section{Education Attainment, Dual Eligibility, and Hospital Rating}

In addition, we found a consistent and statistically significant relationship between education attainment and hospital ratings. Compared with counties with high education attainment (reference group), hospitals in counties with intermediate education attainment are more likely to have one-star ratings. Similarly, hospitals in counties with less and intermediate education attainment are less likely to be five-star rated. Consistently, odds ratios of hospitals in intermediate and lower education attainment counties with better quality are significantly lower, at the $1 \%$ level.

In terms of dual eligibility, hospitals in counties with higher percentage of dual-eligible residents are statistically significantly less likely to receive five-star ratings. Consistent evidence was found in odds ratios. However, dual eligibility is not 
TABLE 1. Distribution of Hospital Overall Quality Rating by Socioeconomic and Geographic Factors

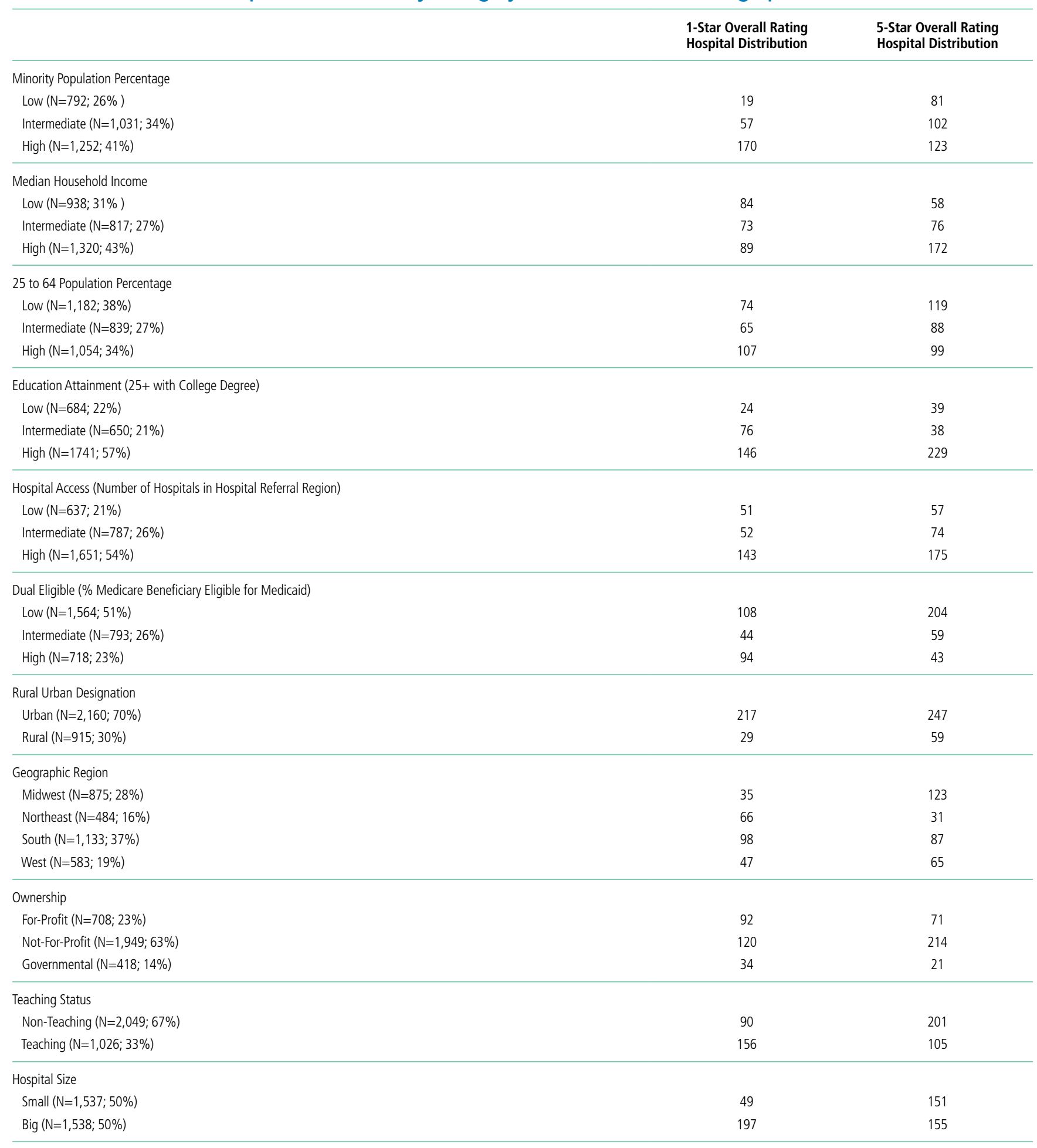

statistically significantly correlated with the probabilities of receiving one-star ratings.

Rurality, Geographic Region, and Hospital Rating Compared with urban hospitals, rural hospitals are less likely to receive five-star ratings. However, there is no difference in the probabilities of receiving one-star ratings and no statistically significant difference in overall ratings. Geographically, hospitals in the Northeast are more likely to have onestar ratings and less likely to be five-star rated. The odds ratio also suggests that Northeastern hospitals on average have lower quality rating compared with Midwestern hospi- 
TABLE 2. Marginal Probabilities and Odds Ratios by Socioeconomic and Geographic Factors

\begin{tabular}{|c|c|c|c|}
\hline & \multicolumn{2}{|c|}{ Marginal Effects } & \multirow{2}{*}{$\begin{array}{c}\text { Odds Ratios } \\
\text { Star Rating (1-5 Scale) }\end{array}$} \\
\hline & 1-Star Rating & 5-Star Rating & \\
\hline \multicolumn{4}{|c|}{ Minority Population Percentage (ref: Low Minority Population Percentage Group) } \\
\hline Intermediate & 0.03 & $-0.02^{*}$ & $0.72^{* *}(0.58,0.90)$ \\
\hline High & $0.08^{* *}$ & 0.00 & $0.52^{* *}(0.40,0.69)$ \\
\hline \multicolumn{4}{|c|}{ Household Income (ref: High Income Group) } \\
\hline Low & $0.04 *$ & 0.00 & $0.67^{*}(0.49,0.91)$ \\
\hline Intermediate & $0.03^{*}$ & -0.01 & $0.76^{*}(0.60,0.98)$ \\
\hline \multicolumn{4}{|c|}{25 to 64 Population Pct (ref: High 25 to 64 Population Percentage Group) } \\
\hline Low & -0.01 & 0.01 & $1.05(0.85,1.29)$ \\
\hline Intermediate & -0.01 & 0.01 & $1.07(0.86,1.34)$ \\
\hline \multicolumn{4}{|c|}{ Education Attainment (ref: High Education Attainment Group) } \\
\hline Low & 0.01 & $-0.04^{*}$ & $0.66^{* *}(0.51,0.85)$ \\
\hline Intermediate & $0.07^{* *}$ & $-0.04^{*}$ & $0.53^{* *}(0.41,0.67)$ \\
\hline \multicolumn{4}{|c|}{ Access (ref: High Number of Hospitals in Referral Region Group) } \\
\hline low & 0.00 & -0.01 & $1.10(0.87,1.39)$ \\
\hline Intermediate & -0.02 & 0.00 & $1.05(0.83,1.33)$ \\
\hline \multicolumn{4}{|c|}{ Dual Eligible (ref: Low Dual Eligible Percentage Group) } \\
\hline Intermediate & -0.03 & $-0.02^{*}$ & $0.97(0.78,1.21)$ \\
\hline High & 0.02 & $-0.02^{* *}$ & $0.77^{*}(0.59,1.00)$ \\
\hline \multicolumn{4}{|c|}{ Rural-Urban Designation (ref: Urban Hospitals) } \\
\hline Rural & -0.02 & $-0.03^{* *}$ & $1.09(0.89,1.34)$ \\
\hline \multicolumn{4}{|c|}{ Geographic Region (ref: Midwest) } \\
\hline Northeast & $0.09^{* *}$ & $-0.05^{* *}$ & $0.37^{* *}(0.25,0.56)$ \\
\hline South & 0.01 & $-0.03^{* *}$ & $0.68^{*}(0.51,0.91)$ \\
\hline West & 0.03 & $-0.02^{*}$ & $0.69^{*}(0.49,0.97)$ \\
\hline
\end{tabular}

Columns (1) and (2) report the marginal effects in probabilities of being 1-Star or 5-Star hospitals. The results are estimated using logistic regression.

Column (3) reports the odd ratios based on multinominal logistic regression, where the outcome variable is an ordinal star rating from 1 to 5.

All regressions also adjust for hospital ownership, teaching status, hospital size, and the number of hospitals available in each Hospital Referral Region.

${ }^{*}$ and ${ }^{* *}$ denote statistically significant at $5 \%(P<0.05)$ and $1 \%(P<0.01)$ levels.

Standard errors are clustered at Hospital Referral Regions.

$\mathrm{Cl}=95 \%$ confidence interval

Odds ratios of the multinomial logistic regression are produced by the cumulative probability. The result shows the odds of being rated higher related to a specific level of the categorical predictor controlling for other predictors.

tals. Hospitals in South and West are also less likely to have five-star ratings.

\section{DISCUSSION}

Consistent with findings in nursing homes, ${ }^{10}$ hospitals that serve lower income communities have comparatively lower quality ratings than did those that serve more affluent communities. Several factors may contribute to these outcomes. Higher volumes of uninsured patients and patients with public insurance impact how much revenue the hospital collects for services, hindering the capacity to reinvest in processes to advance quality. Moreover, these hospitals are likely to serve patients with higher acuity and complex psychosocial barriers that affect their experience, perceptions, and outcomes. Structural conditions of economically distressed communities also play a role. Limited access to a robust network of community-based resources for healthy living post surgery may contribute to higher rates of readmission, which may compromise overall quality ratings.
Furthermore, after adjustment for community characteristics, hospitals that serve higher volumes of racial minorities have higher probability of receiving one-star ratings and lower average quality rating. While more research is needed to examine specific measures in the quality rating formula that may disproportionately affect racial and ethnic minorities, Hospital Consumer Assessment of Healthcare Providers and Systems (HCAHPS) surveys may offer some insight. Some researchers have found that White respondents and those with higher levels of education are more likely to cite favorable HCAHPS responses than are minorities or persons with lower levels of education. ${ }^{11}$ This has negative implications on the HCAPHS scores of hospitals that serve higher volumes of minority patients with low education attainment. Real or perceived discrimination, unconscious bias, miscommunication, and language discordance may explain the disparity between the survey results of White respondents and minorities. ${ }^{12-16}$ 
While interpreting the results of this study, it is important to note that the research design examines the relationship between quality ratings, race, and community characteristics. Our analysis does not specifically examine clinical quality of care. It should not be assumed that hospitals with low ratings provide substandard clinical care.

While the intent of Hospital Quality Ratings is well received, there are varying perspectives on the calculation methodology-particularly the need for social risk adjustment. ${ }^{17-19}$ There is also concern about community perception which affects consumer choice, decision making, and referral patterns. Hospitals with lower ratings are likely to have negative repercussions that perpetuate inequities. For example, in light of new and emerging pay-for-performance models, the publicity of star ratings has the potential to influence behaviors that exacerbate disparities. ${ }^{20}$ Physicians and medical groups may explicitly or implicitly avoid patients with characteristics that may lower their quality scores. Patients with resources to fully cover their healthcare expenses may choose hospitals with higher quality ratings, leaving hospitals with lower quality ratings to serve the under- or uninsured. Over time, these patterns may jeopardize quality, safety, and the fiscal viability of hospitals that serve communities with lower socioeconomic status.

Among the geographic regions analyzed, quality ratings were higher in the Midwest. This finding aligns with a report from the Agency for Healthcare Research and Quality, which recognized five states from the Midwest for having the highest quality ratings (lowa, Minnesota, Nebraska, North Dakota, and Wisconsin). ${ }^{21}$ Hospitals in the South and Northeast generally had lower quality ratings. As discovered by other investigators, nonteaching, smaller, rural hospitals had more favorable outcomes when compared with teaching, larger, urban hospitals, which are more likely to care for more complex, critically ill patients. ${ }^{22}$ These regional differences, coupled with hospital types, have implications for federal appropriations and funding priorities earmarked for quality initiatives.

\section{CONCLUSION}

As national efforts continue to promote health equity and enhance the value of healthcare, it is important to recognize the association between race, socioeconomic factors, and hospital star quality ratings. Allocated resources should ensure that hospitals serving racial minorities, low-income communities, and those in urban settings have the capacity to deliver comprehensive care based on the unique needs of the community. Hospitals that serve low-income communities may benefit from payment models and incentives that adjust for these differences - which could allow them to invest in quality improvement processes and social support services.

Disclosures: The authors declared no potential conflicts of interest with respect to the research, authorship, and/or publication of this article.

Funding: The authors did not receive external funding for this study.

\section{References}

1. Statistica. U.S. Hospitals - Statistics \& Facts. www.statista.com. Accessed May 22, 2019. https://www.statista.com/topics/1074/hospitals/

2. Centers for Medicare \& Medicaid Services. Hospital Compare overall hospital rating. Accessed May 22, 2019. https://www.medicare.gov/hospitalcompare/Data/Hospital-overall-ratings-calculation.html

3. DeLancey JO, Softcheck J, Chung JW, Barnard C, Dahlke AR, Bilimoria KY. Associations between hospital characteristics, measure reporting, and the Centers for Medicare \& Medicaid Services Overall Hospital Quality Star Ratings. JAMA. 2017;317(19):2015-2017. https://doi.org/10.1001/jama.2017.3148

4. Osborne NH, Upchurch GR, Mathur AK, Dimick JB. Explaining racial disparities in mortality after abdominal aortic aneurysm repair. J Vasc Surg. 2009;50(4):709-713. https://doi.org/10.1016/j.jvs.2009.05.020

5. Trivedi AN, Sequist TD, Ayanian JZ. Impact of hospital volume on racial disparities in cardiovascular procedure mortality. J Am Coll Cardiol. 2006;47(2):417-424. https://doi.org/10.1016/j.jacc.2005.08.068

6. Hu J, Nerenz D. Relationship between stress rankings and the overall hospital star ratings: an analysis of 150 cities in the United States. JAMA Intern Med. 2017;177(1):136-137. https://doi.org/10.1001/jamainternmed.2016.7068

7. Herrin J, Andre JS, Kenward K, Joshi MS, Audet AM, Hines SC. Community factors and hospital readmission rates. Health Serv Res. 2015;50(1):20-39. https://doi.org/10.1111/1475-6773.12177

8. Brewster AL, Lee S, Curry LA, Bradley EH. Association between community social capital and hospital readmission rates. Popul Health Manag. 2018;22(1):40-47. https://doi.org/10.1089/pop.2018.0030

9. Navathe AS, Zhong F, Lei VJ, et al. Hospital readmission and social risk factors identified from physician notes. Health Serv Res. 2018;53(2):1110-1136. https://doi.org/10.1111/1475-6773.12670

10. Yuan Y, Louis C, Cabral H, Schneider JC, Ryan CM, Kazis LE. Socioeconomic and geographic disparities in accessing nursing homes with high star ratings. J Am Med Dir Assoc. 2018;19(10):852-859.e2. https://doi.org/10.1016/j.jamda.2018.05.017

11. Goldstein E, Elliott MN, Lehrman WG, Hambarsoomian K, Giordano LA. Racial/ethnic differences in patients' perceptions of inpatient care using the HCAHPS survey. Med Care Res Rev. 2010;67(1):74-92. https://doi. org/10.1177/1077558709341066

12. Jacobs EA, Rathouz PJ, Karavolos K, et al. Perceived discrimination is associated with reduced breast and cervical cancer screening: the study of women's health across the nation (SWAN). J Womens Health (Larchmt). 2014;23(2):138-145. https://doi.org/10.1089/jwh.2013.4328

13. Reskin B. The race discrimination system. Annu Rev Sociol. 2012;38(1):17-35. https://doi.org/10.1146/annurev-soc-071811-145508

14. Chapman EN, Kaatz A, Carnes M. Physicians and implicit bias: how doctors may unwittingly perpetuate health care disparities. J Gen Intern Med. 2013;28(11):1504-1510. https://doi.org/10.1007/s11606-013-2441-1

15. DeVoe JE, Wallace LS, Fryer Jr GE. Measuring patients' perceptions of communication with healthcare providers: do differences in demographic and socioeconomic characteristics matter? Health Expect. 2009;12(1):70-80. https://doi.org/10.1111/j.1369-7625.2008.00516.x

16. Austin JM, Jha AK, Romano PS, et al. National hospital ratings systems share few common scores and may generate confusion instead of clarity. Health Aff (Millwood). 2015;34(3):423-430. http://doi.org/10.1377/hlthaff.2014.0201

17. Halasyamani LK, Davis MM. Conflicting measures of hospital quality: Ratings from "Hospital Compare" versus "Best Hospitals." J Hosp Med. 2007;2(3):128-134. https://doi.org/10.1002/jhm.176

18. Lavenberg JG, Leas B, Umscheid CA, Williams K, Goldmann DR, Kripalani $\mathrm{S}$. Assessing preventability in the quest to reduce hospital readmissions. J Hosp Med. 2014;9(9):598-603. https://doi.org/10.1002/jhm.2226

19. Bilimoria $K Y$, Barnard $C$. The new $C M S$ hospital quality star ratings: the stars are not aligned. JAMA. 2016;316(17):1761-1762. https://doi.org/10.1001/ jama.2016.13679

20. Casalino LP, Elster A, Eisenberg A, Lewis E, Montgomery J, Ramos D. Will pay-for-performance and quality reporting affect health care disparities? Health Aff (Millwood). 2007;26(3):w405-w414. https://doi.org/10.1377/ hlthaff.26.3.w405

21. Agency for Healthcare Research \& Quality. Overview of Quality and Access in the U.S. Health Care System. Published July 3, 2017. Accessed May 23, 2019. https://www.ahrq.gov/research/findings/nhqrdr/nhqdr16/overview.html

22. Wang DE, Tsugawa Y, Figueroa JF, Jha AK. Association between the Centers for Medicare and Medicaid Services hospital star rating and patient outcomes. JAMA Intern Med. 2016;176(6):848-850. https://doi.org/10.1001/ jamainternmed.2016.0784 